\title{
Cadernos abertos de laboratório e publicações líquidas: novas tecnologias literárias para uma Ciência Aberta
}

\author{
Open notebook science and liquid publications: elements to build up a \\ new epistemic culture in the Open Science movement
}

\section{Cuaderno abierto de laboratório y publicaciones líquidas: elementos para la construcción de una nueva cultura epistemica en el movimiento por la Ciencia Abierta}

\author{
Anne Clinio | anneclinio@gmail.com \\ Instituto Brasileiro de Informação em Ciência e Tecnologia. Rio de Janeiro, Brasil. \\ Universidade Federal do Rio de Janeiro. Rio de Janeiro, Brasil. \\ Sarita Albagli | sarita.albagli@gmail.com \\ Instituto Brasileiro de Informação em Ciência e Tecnologia. Rio de Janeiro, Brasil. \\ Universidade Federal do Rio de Janeiro. Rio de Janeiro, Brasil.
}

\section{Resumo}

Apresenta resultados de pesquisa sobre o conceito e práticas de caderno aberto de laboratório, inovação criada no âmbito do movimento contemporâneo por uma Ciência Aberta. O arcabouço teórico adota a noção de cultura epistêmica e a perspectiva das "três tecnologias" de Shapin e Shaffer enquanto, no campo empírico, adentramos o "laboratório aberto" através de pesquisa documental. Identificamos que essa inovação não é uma melhoria incremental, mas uma nova "tecnologia literária" para um novo modo de produzir e comunicar ciência. Ela dialoga com a noção de publicação líquida e é um elemento estratégico de um ecossistema de colaboração aberta que pretende, entre outras coisas, substituir uma "ciência baseada na confiança" por uma ciência fundamentada na transparência e na proveniência dos dados. Articulada com novas tecnologias materiais e sociais, fomenta uma cultura epistêmica que chamamos de matter of proof por valorizar, sobretudo, a habilidade dos cientistas em documentar adequadamente os experimentos que subsidiam suas afirmações.

Palavras-chave: Open notebook science; caderno aberto de laboratório; Ciência Aberta, Jean-Claude Bradley; cultura epistêmica; matter of proof; publicação líquida. 


\begin{abstract}
Reports results research project on the concept and practices of open notebook science, an innovation created in the context of the contemporary Open Science movement. Our theoretical framework adopts the notion of epistemic culture and the "three technologies" perspective of Shapin and Shaffer while our empirical research enters the "open laboratory" through a documentary research. The research identifies that this innovation is not an incremental improvement, but a new "literary technology" that fosters a new way of producing and communicating science. It dialogues with the liquid publications notion and it is a strategic element of a complex, open collaborative ecosystem that aims to move from a "science based on trust" to a science based on transparency and data provenance. Embedded within new material and social technologies, it fosters a epistemic culture that we name matter of proof because it values, above all, the ability of scientists to adequately document the experiments that subsidize their claims.
\end{abstract}

Keywords: Open notebook science; Open Science; Jean-Claude Bradley; epistemic culture; matter of proof; liquid publications.

\title{
Resumen
}

Presenta resultados de investigación de doctorado sobre el concepto y prácticas de open notebook science, innovación creada en el contexto del movimiento contemporáneo por una Ciencia Abierta. El marco teórico adopta la noción de cultura epistémica y la perspectiva de las "tres tecnologías" de Shapin y Shaffer mientras que la investigación empírica adentra el "laboratorio abierto" a través de investigación documental. El estudio identifica que la innovación no es una mejora incremental, sino más bien una nueva "tecnología literaria" que fomenta un nuevo modo de producir y comunicar ciencia. Ella dialoga con la noción de "publicación líquida" y es el principal elemento de un complejo ecosistema de colaboración abierta que pretende realizar el tránsito de una "ciencia basada en la confianza" a una ciencia basada en la transparencia y la procedencia de los datos. Articulada con nuevas tecnologías materiales y sociales, fomenta una cultura epistemica que llamamos matter of proof por valorar, sobretodo, la habilidad de los científicos en documentar adecuadamente los experimentos que subsidian sus afirmaciones.

Palabras-clave: Open notebook science; cuaderno abierto de laboratorio; Ciencia Abierta; Jean-Claude Bradley; cultura epistemica; matter of proof; publicación líquida. 


\section{Introdução}

Este artigo apresenta resultados de uma pesquisa ${ }^{i}$ cujo objetivo é demonstrar como a proposta de caderno aberto de laboratório (open notebook science, na versão original em inglês) é parte de uma nova cultura epistêmica ${ }^{1}$ que está sendo engendrada no âmbito do movimento contemporâneo da Ciência Aberta. Trata-se de uma inovação no modo de produzir e comunicar ciência, desenvolvida por diversos cientistas, entre os quais destacamos Jean-Claude Bradley, professor e pesquisador de Química na Universidade de Drexel University. Bradley foi o principal entusiastaii desta iniciativa que pretende "promover um debate mais consistente sobre colaboração aberta em ciência"².

A pesquisa trabalha a partir de dois eixos teórico-metodológicos: a noção de cultura epistêmica e a perspectiva das três tecnologias de Shapin e Shaffer ${ }^{3}$. A noção de cultura epistêmica se refere às "culturas que criam e certificam o conhecimento", evidenciando a fragmentação da ciência em diferentes monopólios epistêmicos independentes e contrariando a ideia de unicidade e uniformidade nas práticas científicas, inclusive nas ciências naturais. Uma nova cultura epistêmica expressa "amálgamas de arranjos e mecanismos alcançados - ligados pela afinidade, necessidade e coincidência histórica - que, em um determinado campo, compõem como nós sabemos o que sabemos"1, e ainda as "conjunções de convenções e dispositivos parcialmente elaborados, organizados e dinamizados sobre os quais nenhum ator individual tem controle" . Desse ponto de vista, os cadernos abertos de laboratório constituem uma prática resultante desses arranjos e não apenas produto intelectual ou tecnológico.

Por outro lado, o estudo adota a perspectiva das três tecnologias, elaborada por Shapin e Shaffer para compreender o processo de legitimação e consolidação de uma nova cultura epistêmica no alvorecer da Ciência Moderna pela articulação exitosa de três tecnologias - material, social e literária -, que estabeleceu a noção de fato científico como "variedade de conhecimento" tão sólida que se tornou "o fundamento do conhecimento apropriado" e sinônimo da própria ciência.

No plano empírico, partindo da premissa do próprio Jean-Claude Bradley de que o caderno aberto de laboratório, na sua forma ideal, elimina a assimetria da informação entre pesquisadores e o resto do mundo, adentramos seu "laboratório aberto" por meio de extensa pesquisa documentaliii. Seus relatos sobre o processo de abertura dos cadernos de laboratório nos permitem identificar como as três tecnologias de Shapin e Shaffer estão sendo articuladas para criar uma nova cultura epistêmica que chamamos de matter of proof (questão de prova) pela ênfase dada à documentação dos experimentos. A escolha dessa expressão visa contrastar com o matter of fact (fato científico) da Ciência Moderna, que valoriza, sobretudo, a construção do fato científico.

\footnotetext{
i Pesquisa realizada por Anne Clinio no âmbito do PPGCI Ibict UFRJ, intitulada "Novos cadernos de laboratório e novas culturas epistêmicas: entre as políticas do experimento e o experimento da política", sob orientação da professora Sarita Albagli e coorientação do Dr. Antonio Lafuente, do Consejo Superior de Investigaciones Cientificas (Csic). A pesquisa contou com apoio da Fundação de Amparo à Pesquisa do Estado do Rio de Janeiro (Faperj) e da Coordenação de Apoio ao Pessoal do Ensino Superior (Capes).

ii Consideramos Jean-Claude o principal "recrutador" de apoios para o conceito e práticas do open notebook science por sua atuação diferenciada. No entanto, é preciso citar a participação de Andrew Lang, Bill Hooker, Cameron Neylon, Rajarshi Guha, Steve Koch, Anthony Williams, Matthew Todd, Anthony Salvagno, Rajarshi Guha, Philip Rosenthal Daniel Zaharevitz, entre outros early adopters e colaboradores.

iii As principais fontes de informação são os blogs UsefulChem and Drexel CoAS E-Learning; seu caderno aberto de laboratório Open Notebook Science (wiki), artigos, capítulos de livros, entrevistas, palestras publicadas em seu canal no YouTube. áudios da disciplina "Recuperação de Informação em Química" e apresentações eletrônicas publicadas na plataforma Slide Share.
} 


\section{As origens e os argumentos a favor do caderno aberto de laboratório}

Segundo Jean-Claude Bradley4, o caderno aberto de laboratório, é "uma maneira de fazer ciência na qual - da melhor maneira possível - você torna toda a sua pesquisa livre e acessível ao público em tempo real". Esta proposta reivindica uma nova forma de comunicação formal entre cientistas, que seria aprimorada e acelerada pela abertura precoce de informações e resultados de pesquisa. Para tal, propõe novas práticas como a disponibilização em tempo real da íntegra dos registros dos cadernos de laboratório em plataformas on-line e a adoção de licenças livres que regulamentam o acesso, a reutilização e a redistribuição de conteúdo por qualquer pessoa ou sistema automatizado. $\mathrm{O}$ autor afirma que:

[...] Para aclarar a confusão, eu vou utilizar a expressão open notebook science, que ainda não sofreu uma mutação memética. Com ela, eu me refiro à existência de uma URL linkada a um caderno de laboratório (como este) que está disponível abertamente e é indexado por ferramentas de busca habituais. Ele não precisa obrigatoriamente parecer com um caderno de laboratório de papel, mas é essencial que as informações necessárias para que o pesquisador chegue às suas conclusões estejam igualmente disponíveis para o resto do mundo. Basicamente, nenhuma informação privilegiada. ${ }^{\text {iv }}$

A origem da proposta de cadernos abertos de laboratório se situa, por um lado, em um conjunto de críticas sobre os modos de produção e comunicação do conhecimento científico, incluindo a rejeição da própria noção de fato científico, e, por outro lado, na identificação de oportunidades pela confluência de novas formas de colaboração em ambientes digitais e o surgimento de uma cultura digital livre.

Segundo Bradley, o modo dominante de comunicação formal do conhecimento científico, baseado majoritariamente na publicação de artigos científicos em revistas com sistema de avaliação às cegas por pares, afeta negativamente a pesquisa científica, pois fomenta uma cultura do segredo entre pesquisadores, desestimula a colaboração, promove informação de qualidade duvidosa, ao mesmo tempo que elimina informação relevante, desperdiça recursos financeiros e humanos, gera retrabalho e retarda o desenvolvimento científico. $\mathrm{O}$ autor reconhece que, no passado, a revisão anônima por pares era o mecanismo possível para resguardar, em alguma medida, a qualidade das contribuições e evitar o desperdício de tempo de pesquisadores em fontes de informação cujo retorno não era claro ou garantido5. No entanto, o autor advoga que, após a popularização das tecnologias digitais, é necessário o estabelecimento de novos critérios e processos de revisão por pares - entre eles, a rápida disponibilização on-line dos resultados da pesquisa, bem como a qualidade da descrição dos experimentos. Isto porque, entre outros fatores, identifica a internet como oportunidade tecnológica para eliminar a mediação da comunicação do conhecimento científico em termos editoriais, técnicos e jurídicos.

Bradley ressalta que a maioria dos artigos científicos de sua área oferece descrições sobre os procedimentos de um experimento de forma altamente condensada, genérica e insuficiente para sua reprodução, mesmo que por um outro cientista relativamente competente ${ }^{6}$. Tais lacunas de informação são, segundo ele, resultado da mediação da comunicação científica por um grupo muito pequeno de atores que atua como gatekeepers da informação. São eles que definem temas prioritários, formatos, critérios de cientificidade e qualidade, enfim, o que é publicável em revistas científicas. Na sua visão, a revisão às cegas por pares é "uma maneira rentável de manter a qualidade e foco de uma revista" que atende apenas aos interesses particulares de três segmentos de uma ampla comunidade científica: 1) o editor de periódico científico, que analisa a aderência dos trabalhos à linha editorial da publicação ambicionando torná-la referência em uma área de conhecimento; 2) o autor, em seu desejo de publicizar um trabalho e obter crédito por

iv Tradução das autoras. 
suas contribuições em um campo de conhecimento; 3) os avaliadores, que analisam os trabalhos sem a necessidade de reproduzir os experimentos que respaldam as afirmações dos autores.

Neste sentido, Bradley questiona a qualidade das revistas científicas, incluindo as mais célebres, pela sua limitada capacidade de avaliação - restrita a dois ou três avaliadores anônimos que emitem pareceres de maneira voluntária e precária, em um curto espaço de tempo, sem a possibilidade de reproduzir experimentos e tendo que lidar com as lacunas de informação relevante por parte dos autores. Na contramão, vislumbra o caderno aberto de laboratório como uma plataforma de revisão aberta por pares que ampliaria a escala do processo porque:

As únicas pessoas verdadeiramente qualificadas para avaliar uma parte de uma pesquisa são aquelas que efetivamente analisem os dados brutos para ver se tudo se congrega e isso leva tempo, admitindo que eles têm acesso. É improvável que alguém faça tal coisa sem estar devidamente motivado - geralmente apenas pesquisadores tentando reproduzir um experimento para seus próprios interesses têm uma boa razão para investir esse tempo. ${ }^{8}$

Sua crítica sobre o processo editorial também recai sobre a questão quantitativa, ou seja, o volume de informação relevante (não) comunicado formalmente entre pares. O autor estimou que, segundo os critérios das revistas científicas, $87 \%$ da sua produção científica não ultrapassam os muros de seu laboratório por se tratar de experimentos que não alcançam os "resultados esperados", sendo, portanto, classificados como "fracassados" e irrelevantes para publicação. Bradley discorda dessa lógica que, segundo ele, fomenta nos pesquisadores a obsessão pela construção de um fato científico e a procrastinação na comunicação de resultados de pesquisa. Na contracorrente, ele fomentou o compartilhamento precoce de informação sobre experimentos, independente do estágio dos resultados obtidos, entendendo que a ciência não é feita só de "êxitos", mas também daquilo que equivocadamente é chamado de "falha":

Há uma enorme quantidade de informação útil sobre reações ou tentativas de reações que nunca é compartilhada. Independentemente da obtenção ou não de uma reação "bem sucedida", se sua execução é cuidadosamente registrada, ela pode fornecer informações valiosas. Existem algumas ferramentas e padrões excelentes que permitem, de maneira fácil, realizar uma marcação semântica de reações e de propriedades químicas, de modo que um experimento pode ser disponibilizado para descoberta assim que é iniciado. 9

E ainda:

[...] Qualquer estudante de química pode relatar o enorme valor em discutir experiências fracassadas com pessoas que são tão ou mais bem informadas. No entanto, este debate se limita geralmente aos colegas do laboratório. Ao registrar as experiências em andamento em um blog, eu posso ajudá-lo simplesmente por saber o que você está tentando realizar mesmo, que você ainda não tenha conseguido. ${ }^{10}$

Vale destacar que sua reivindicação pela abertura do conhecimento científico não se limita ao compartilhamento aberto de dados brutos e informações sobre experimentos considerados "bem sucedidos", logo mais favoráveis à reputação de pesquisadores. Pelo contrário, o autor demanda o compartilhamento de todas as informações e dados associados a um experimento, seja qual for o seu status (em andamento, finalizado, abandonado) ou resultado (favorável, desfavorável, contraditório ou ambíguo). Isto porque, no seu entendimento, compartilhar, nos estágios intermediários, incertezas e dificuldades da pesquisa científica é a melhor estratégia para atrair colaboradores e recursos, segundo o mote de que "ciência mais 
rápida, ciência melhor" (fast science, better science). Ao lançar o projeto UsefulChem ${ }^{v}$ em 2005, o seu objetivo era "realizar uma contribuição útil para a sociedade"vi que estava sendo frustrada, em parte, por uma "cultura do segredo"

Uma grande falha no atual sistema de publicação científica é que ainda há demasiada confiança. Espera-se que os leitores confiem nos editores para selecionar pares anônimos e adequados para avaliar as submissões. Os avaliadores confiam nos autores principais que relatam de maneira resumida os resultados de pesquisa. Os autores principais confiam em seus colaboradores, estudantes e pós-doutores na produção de informação precisa para os artigos. Se tornamos públicos o caderno de laboratório e os dados brutos associados a uma pesquisa, podemos reduzir significativamente a quantidade de confiança necessária para manter este castelo de cartas em pé. ${ }^{12}$

Nesse sentido, a disponibilização aberta dos dados de pesquisa por meio de cadernos abertos de laboratório potencializa as oportunidades de escrutínio, validação, correção, refutação, complementação, colaboração e aprendizagem por uma audiência mais ampla, pois:

Não há nenhum gatekeeper a ser convencido neste sistema. Nenhum software para fazer download. Nenhum servidor a ser configurado. Praticamente nenhuma curva de aprendizagem. Qualquer um que faça ciência é livre para replicar em seu campo de interesse. Ciência plenamente democrática. ${ }^{10}$

O autor vislumbra que esta inovação também combate o "efeito de cascata produzido por fontes confiáveis" (trusted source cascade effect), criado pelo mau hábito dos pesquisadores de considerar uma informação insuspeita por ter sido publicada em um periódico científico. Ao declarar que "não existe fonte confiável", Bradley assinala que a ilusão da confiabilidade gera um uso indiscriminado, e muitas vezes inadequado, de resultados de pesquisa. Nesse sentido, advoga pela substituição de uma cultura de "confiança em uma autoridade" (trust in an authority), baseada em "fontes confiáveis", para uma cultura de "desconfiança de tudo e de todos" (mistrust of everything and everybody) que fomenta novas práticas baseadas na transparência, na qualidade das evidências e na preservação da proveniência de dados (data provenance). Ou seja, no histórico sobre o conjunto dos dados que permite rastrear a origem, as transformações pelas quais foram submetidos, as análises e as interpretações realizadas pelos cientistas ao longo da pesquisa.

Além disso, considera o fato científico como uma noção problemática que distorce a comunicação formal entre cientistas ao gerar a percepção de que expor dados ambíguos ou contraditórios é prejudicial para o pesquisador. Com a frase "não existem fatos em ciência, apenas mensurações às quais são incorporadas suposições” ${ }^{13}$, o autor indica que os dados resultantes de experimentos não são irrefutáveis. No melhor dos casos, são evidências sobre as quais os pesquisadores trabalham com algum grau de incerteza e sobre as quais várias suposições são feitas durante o processo de interpretação.

Em suma, o caderno aberto de laboratório documenta de forma aberta, on-line e em tempo real as atividades de pesquisa com o objetivo de fomentar a colaboração aberta entre pesquisadores. Esta inovação aproxima-se do modelo de comunicação de translação ${ }^{14}$, pois torna visíveis as estratégias utilizadas pelos pesquisadores para alistar e manter sob controle o grande número de associações necessárias entre humanos (cientistas, engenheiros, financiadores, administradores públicos, cidadãos etc.) e não-humanos (literatura especializada, laboratório, máquinas, entre outros), até a relativa estabilização dos resultados de

\footnotetext{
v Inciativa com objetivo de sintetizar compostos antimaláricos; congrega uma wiki e um blog homônimos que disponibilizam, respectivamente, seu caderno de laboratório e suas reflexões. Disponível em http://usefulchem.blogspot.com.

vi Posteriormente, o projeto se dedica ao desenvolvimento aberto de compostos antimaláricos.
} 
pesquisa. No entanto, vale destacar que se trata também de uma plataforma de revisão por pares aberta (a todos e todas), transparente (a vista de todos), contínua (sem data de expiração), interativa (admite réplicas e tréplicas), dinâmica (com novos comentários e retificações ao longo do tempo) e "deseditorializada" (sem controle prévio de um comitê editorial). Para Bradley, essa é a maneira mais produtiva de lidar com as ambiguidades intrínsecas à prática científica que, embora não possam ser completamente eliminadas, podem ser reduzidas por meio de novas dinâmicas.

Além da questão da produção do conhecimento propriamente dito, essa inovação repercute sobre a questão da sobreposição dos sistemas de comunicação e de crédito de autoria na ciência. Na medida em que a revisão por pares se tornou "uma espécie de padrão de ouro na promoção acadêmica e obtenção de cargos pela contabilidade de publicações"5, o número de artigos científicos publicados é o principal ativo para consolidar as carreiras profissionais dos pesquisadores. Trata-se da síndrome do "publicar ou perecer" que fomenta uma cultura do segredo e estimula a procrastinação no compartilhamento de dados e informações de pesquisa pela necessidade de elaborar narrativas coerentes e persuasivas para convencer avaliadores e editores de que suas pesquisas são relevantes e merecem ser publicadas. O autor discorda dessa lógica e afirma que "ao fazer ciência aberta, a primeira preocupação é a comunicação da informação" e, por isso, afirma que "devemos separar o problema de comunicar de forma eficiente a informação científica do problema de convencer uma comissão sobre o impacto de uma bolsa de estudos para um membro da faculdade" 5 .

Crítica semelhante é feita à revisão às cegas por pares na avaliação de projetos por agências financiadoras. Ademais, considera contraditório que os sistemas de apoio à pesquisa imponham o desenho de projetos que garantam que determinados resultados serão necessariamente obtidos, questionando: "como um empreendimento pelo desconhecido pode afirmar resultados previsíveis?" ${ }^{13}$. No entanto, o autor não sugere a extinção da revisão por pares, mas a necessidade de processos complementares que favoreçam a colaboração e descobertas casuais (serendipitous discoveries) agora facilitadas pela troca de informação através de software livre, bases de dados e outras infraestruturas ${ }^{13}$.

Ao propor mudanças tão significativas, reconhecemos que esta inovação não representa uma simples melhoria incremental dos cadernos de laboratório em seus moldes tradicionais. Trata-se de uma nova tecnologia literária que, mais do que alterar o modo de comunicar o conhecimento científico, pretende fomentar a produção aberta de conhecimento, reestruturar o processo de avaliação por pares, melhorar a qualidade da informação circulante e ampliar a participação na ciência.

\section{A prática do caderno aberto de laboratório: a articulação de "três tecnologias" na composição de uma nova "variedade de conhecimento"}

Quando utilizamos a palavra ciência no singular ou a empregamos sem adjetivos que a qualifiquem, reforçamos a ideia de que a cultura epistêmica habilmente projetada pela Ciência Moderna como "a fundação do conhecimento adequado"3 é a única existente ou possível. Trata-se de um modo de ciência que se caracteriza pela ideia de purificação das instâncias objetivas e subjetivas do conhecimento e pela perspectiva de progresso, entendido como a passagem de um passado arcaico a um futuro melhor. Nesse sentido, falar em ciências, no plural, significa admitir a existência de vários modos de praticá-la.

Pelo exposto, identificamos que o cerne da questão de Jean-Claude Bradley é o que Latour ${ }^{15}$ chama de "modelo de difusão" do conhecimento científico, baseado na comunicação de uma "ciência pronta", publicada no formato de artigos científicos em revistas com sistema de revisão anônima por pares. Segundo Shapin e Shaffer, a origem desta tecnologia literária remonta ao gênero literário dos ensaios experimentais 
(experimental essays), criado por Robert Boyle e os primeiros filósofos experimentais que, no alvorecer da Ciência Moderna, consolidaram uma "nova variedade de conhecimento", até então insólita, como sendo o "próprio espelho da Natureza"3.

No final do século XVII, em um contexto de disputas sobre quais práticas de produção de conhecimento deveriam ser consideradas adequadas para estudar os fenômenos naturais, Boyle e os primeiros filósofos experimentais prescreveram o programa experimental em detrimento de outras opções possíveis, como, por exemplo, a proposta de Thomas Hobbes baseada em noções auto-evidentes que conteriam em si a sua própria fonte de legitimidade. De acordo com os estudos históricos de Shapin e Shaffer, a consolidação do que, à época, representava uma nova cultura epistêmica se deu pela articulação exitosa de três tecnologias:

1. A tecnologia material dos instrumentos científicos e do próprio laboratório como espaço reservado e equipado para a realização de experimentos, cujo objetivo era excluir a interferência humana no estudo dos fenômenos da Natureza;

2. A tecnologia social que determina quem pode produzir conhecimento, como os cientistas devem lidar com as controvérsias e validar conhecimento. Neste caso, adota-se a prática do testemunho por três tipos de testemunhas: (a) as "testemunhas modestas" dos filósofos experimentais que realizavam experimentos e apresentavam as leis por detrás dos fenômenos naturais; (b) as "testemunhas diretas" da seleta plateia de nobres ingleses convidados a assistir presencialmente a execução de experimentos em laboratórios e cuja participação era capitalizada para inferir credibilidade aquilo que foi testemunhado; (c) as "testemunhas diretas, porém distantes" ou "testemunhas virtuais" que "participavam" indiretamente dos experimentos através da leitura dos experimental essays.

3. A tecnologia literária que comunica, torna comum, o fenômeno produzido com auxílio de instrumentos científicos para pessoas que não testemunharam diretamente o experimento. Trata-se do gênero literário dos ensaios experimentais (experimental essays) que prescreve uma série de recomendações estilísticas com o objetivo de projetar na mente do leitor a sensação de simultaneidade e veracidade do experimento que está sendo narrado. Esta operação é auxiliada por representações naturalistas de instrumentos ou processos científicos que preenchem as possíveis lacunas da imaginação dos leitores através de recursos de "assistência visual". Ela é tão crucial para o programa experimental que "a atenção [dada] à escritura de relatos de experimentos era tão importante quanto realizar os próprios experimentos"3

Inspiradas pelo método de análise desenvolvido por Shapin e Shaffer em seus estudos históricos a respeito da polêmica, entre Thomas Hobbes e Robert Boyle, sobre a maneira adequada de conhecer, adotamos a perspectiva das três tecnologias para descrever como a tecnologia literária do caderno de laboratório se articula com novas tecnologias materiais e sociais para engendrar uma nova cultura epistêmica.

\section{A tecnologia material}

O laboratório de Bradley, como outros laboratórios de Química, era equipado com instrumentos científicos, computadores, substâncias químicas, e insumos necessários para a realização de experimentos. Esse computadores eram também utilizados para registrar a pesquisa em cadernos abertos de laboratório, além de dispor de equipamentos para a produção de podcasts com o conteúdo de aulas e captura de imagens de experimentos. Assim como outras unidades de pesquisa, o acesso ao espaço físico do laboratório era controlado, porém acessível a não integrantes da equipe. Nesse sentido, a sua "Química Aberta” não apenas interligaria o já instituído movimento pelo acesso aberto às publicações científicas aos recentes esforços pelo compartilhamento aberto dos dados, como também abrange elementos tangíveis da produção do 
conhecimento. Esta característica propiciou diversas colaborações relevantes ${ }^{\mathrm{vii}}$, destacando-se o primeiro open science loop (maio de 2007) na qual "a elaboração de hipóteses, acoplagem molecular (docking), síntese e os resultados da análise foram realizados de modo aberto". viii

A maneira mais fácil de "entrar" era, porém, acessar seus cadernos abertos. Por isso, nos primeiros anos, Bradley e seus colaboradores investiram fortemente no desenvolvimento de infraestruturas e serviços para a recuperação de informação, tais como a indexação automática de conteúdos por ferramentas de busca não especializadas, como o Google e soluções de RSS que notificavam colaboradores sobre atualizações.

Na prática, seu laboratório aberto conectava uma comunidade científica pequena e descentralizada que reunia especialistas profissionais, amadores, estudantes, técnicos, organizações sem fins lucrativos, empresas comerciais e boots e agregava expertises complementares. Seu "ecossistema de colaboração aberta" compartilhava tecnologias materiais, como amostras de insumos e uso remoto de instrumentos científicos $^{\mathrm{i} x}$, e expandia as possibilidades da pesquisa aberta sobre agentes antimaláricos, pois, em suas palavras, "agora nós não somos apenas um laboratório fazendo pesquisa, mas uma rede de laboratórios que colaboram"16.

\section{A tecnologia literária}

As primeiras práticas do caderno aberto de laboratório utilizaram a plataforma proprietária Blogger para registrar atividades de pesquisa, pois suas funcionalidades cobriam as expectativas iniciais de publicar posts, dialogar com colaboradores via caixa de comentários e oferecer funcionalidades como ferramentas de busca e RSS feed para os leitores. Logo, o autor percebeu que as funcionalidades do blog eram incipientes, pois a colaboração aberta exigia mais do que comentar, sugerir e criticar. Era preciso editar diretamente os conteúdos, reescrever, refazer sem a necessidade de solicitar um login e senha ao administrador da plataforma. O projeto UsefulChem passou então à configuração "bliki” (blog mais wiki), na qual o blog registra o status da pesquisa para um público mais amplo enquanto a wiki documenta a execução de experimentos.

A padronização da "página de experimento" em um formato estruturado cujo preenchimento se torna obrigatório visou garantir a qualidade do registro e possibilitar sua reutilização em serviços de informação. Ela era composta por nove seções: 1) número do experimento; 2) representação gráfica do experimento, 3) nome do pesquisador; 4) objetivo; 5) procedimento; 6) resultado; 7) discussão; 8) conclusão e 9) log.

vii Outras colaborações exitosas são o Open Notebook Science Challenge e o Open Notebook Science Solubility Challenge, projetos de pesquisa crowdsourcing que coletavam medições realizadas por estudantes que adotavam o open notebook science.

viii O open science loop reuniu análises de docking por Rajarshi Guha (Indiana University), síntese de compostos no laboratório de Bradley, testes do grupo de Philip Rosenthal (University of California) e testes de atividade antitumoral oferecidos por Daniel Zaharevitz, chefe do Information Technology Branch of the Developmental Therapeutics Program do National Cancer Institute (NCI).

ix Realizavam experimentos para outros pesquisadores envolvidos na produção aberta de compostos antimaláricos desde que sua equipe aprovasse o plano experimental . 
Figura 1: Experimento n. 269, realizado pelo estudante Matthew McBride

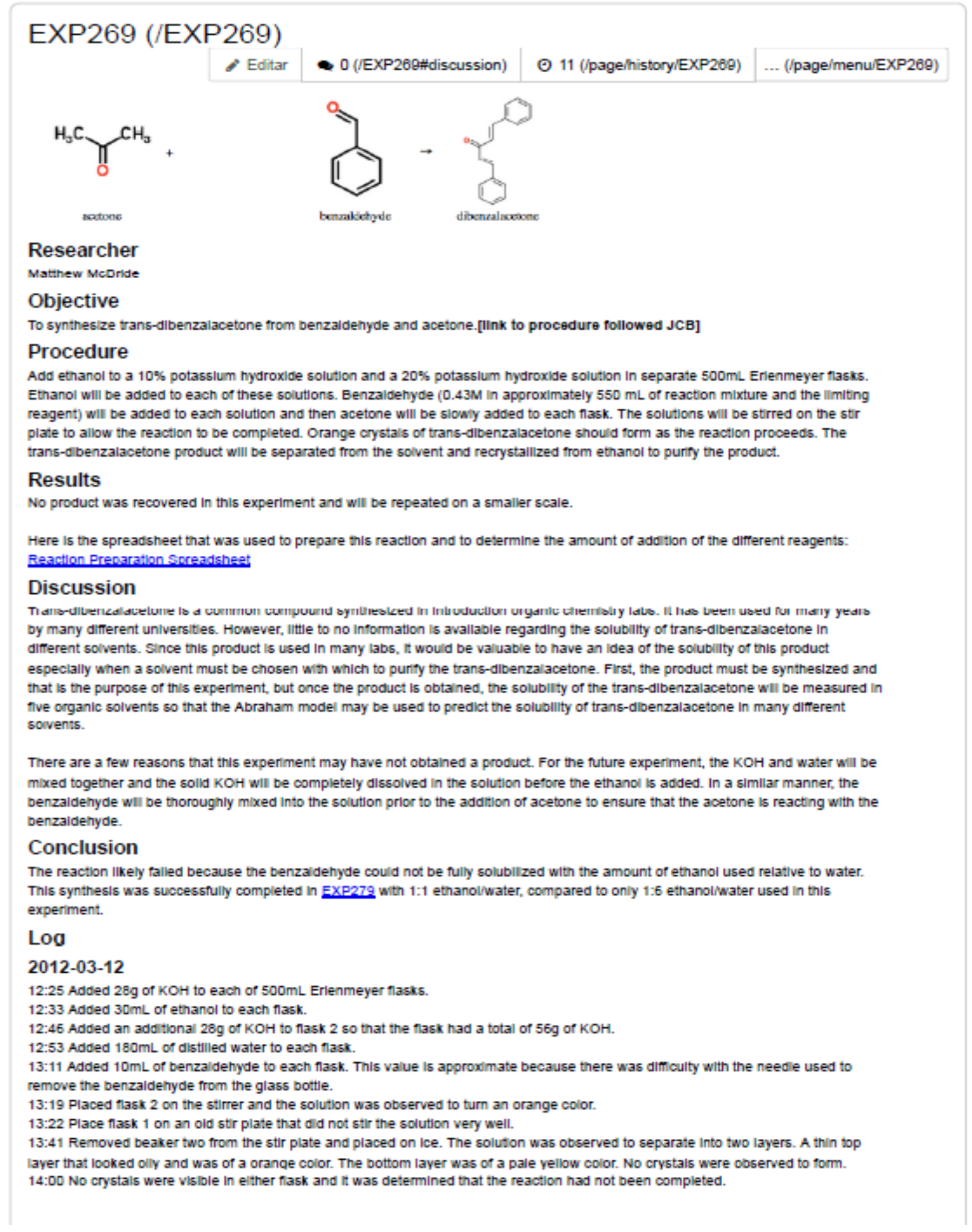

Fonte: Open Notebook Science Challengex

Segundo Bradley, o log (figura 2) é a seção mais importante, pois, no modo ideal, descreve o experimento enquanto ele está sendo realizado - o que garantiria precisão e riqueza de detalhes. A entrada de informações adota um estilo objetivo, sem floreios, no qual o pesquisador relata quem fez o que, onde, como, quando e os resultados parciais obtidos. $\mathrm{O}$ formato dispensa a elaboração de uma narrativa, exigida nos artigos 
científicos e constrói o "experimento sem história" (storyless experiment) ${ }^{17}$, que valoriza os resultados parciais (result-centric).

Figura 2: Detalhe da página de experimento n. 269 - a seção log

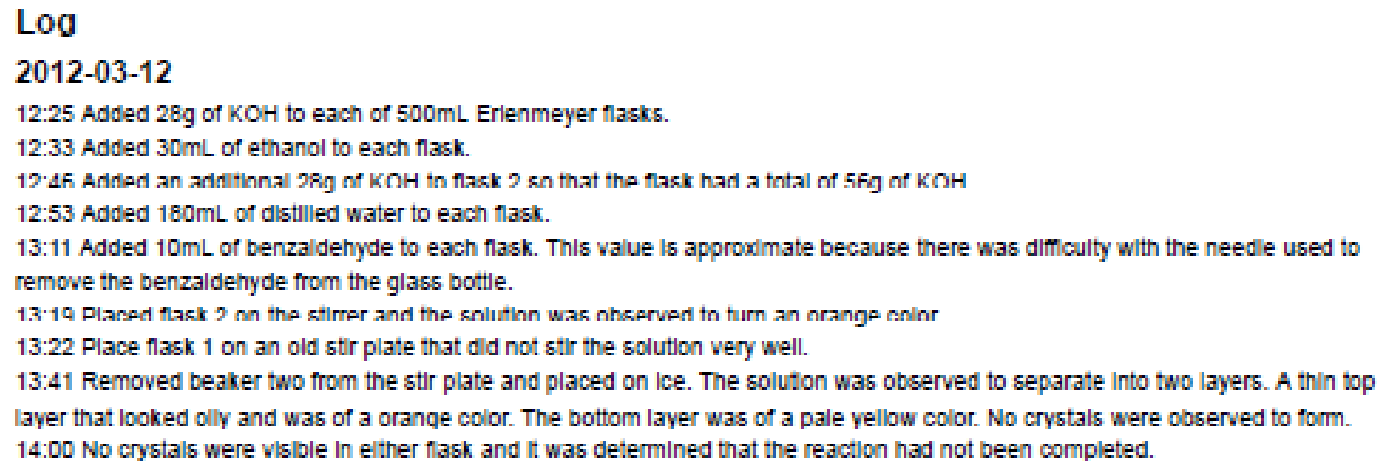

Fonte: Open Notebook Science Challenge ${ }^{\mathrm{xi}}$

Essa concepção se distingue radicalmente das práticas das revistas científicas que privilegiam a comunicação de experimentos (experiment-centric) ou a obtenção de uma molécula (molecule-centric). Sua principal vantagem é que "ao focar em cada resultado de forma independente, não é mais um problema se o objetivo de um experimento foi alcançado ou se um experimento foi abordado em um momento posterior" (UsefulChem, 2012). Com esta inovação, Bradley descarta o critério binário de classificação de um experimento como "bem sucedido" ou "fracassado" pela obtenção (ou não) de um resultado esperado. $\mathrm{Na}$ coleção de registros sobre "Reactions Attempts", por exemplo, tentativas de execução de reações foram reunidas e compartilhadas abertamente porque são consideradas uma oportunidade de estabelecer colaboração entre pesquisadores, estimular excelência ao longo do processo científico e promover discussões mais consistentes em ciência. Nesse sentido, o caderno aberto de laboratório pode funcionar como uma plataforma de rede social (social networking) na medida em que as redes de colaboração se tornam perceptíveis e monitoráveis através da interação dos usuários ${ }^{\mathrm{xii}}$.

Além de novos formatos de arquivo, a prática do caderno aberto demanda infraestruturas. Elas devem ser amigáveis (user friendly), adotar formatos abertos e oferecer funcionalidades para que o seu conteúdo seja referenciável e recuperável por ferramentas de busca, genéricas e especializadas, de modo a criar um "ecossistema de colaboração". Do ponto de vista jurídico, adotou-se a licença Creative Commons Share Alike 2.5 e o grau máximo de abertura processual, disponibilizando a íntegra do conteúdo produzido por seu laboratório em tempo real, sinalizado pelo logo "Conteúdo na Íntegra - Publicação imediata” (All Content - Immediate release) ${ }^{\text {xiii } 18}$. Do ponto de vista técnico, sua experiência com sistemas proprietários de gestão de informação em laboratórios ${ }^{\text {xiv }}$ indicava que a necessidade de aprender um novo software desestimula potenciais colaboradores. Por isso, ele adotou ferramentas web gratuitas amplamente utilizadas. Esta opção também foi vantajosa pela indexação automática e em tempo real do conteúdo por ferramentas

\footnotetext{
xi Disponível em http://onschallenge.wikispaces.com/EXP269 [citado em 2015 ago 10]

xii O doutorando Don Pellegrino analisou o uso da coleção "Reactions Attempts" e identificou redes de colaboração desde os cadernos abertos.
}

xiii Na prática, a maioria dos cadernos de early adopters são parcialmente abertos ou pseudoabertos. Quatro logos, desenvolvidas em 2009, identificam o grau de abertura em termos de omissão ou o adiamento da publicação de dados e informações.

xiv Standard Modular Integrated Research Protocol (SMIRP), a flexible modular collaborative tool that was originally designed to track and manage the dynamic environment of a discovery-driven laboratory research operation. 
de busca como o Google, cujas funcionalidades foram personalizadas para atender às demandas dos colaboradores, tornando o seu conteúdo "facilmente descoberto sem trabalho adicional de formatação". Isto porque "à medida que nos aproximamos de 200 experimentos torna-se claro que a capacidade de recuperar informações é tão importante quanto a de fazer os experimentos"19.

A comunidade de early adopters do caderno aberto de laboratório também investiu na indexação do conteúdo em bancos de dados especializados, como o ChemSpider e X-Ray Crystal Structure Repository. Para tal, a representação da informação adotou formatos machine-readable, com o uso dos identificadores InCh e InChKeys ${ }^{\mathrm{xv}}$. Porém, a indexação do conteúdo resolvia apenas metade do problema, pois também era necessário elaborar questões (queries) para a recuperação dos conteúdos centrados em resultados (result centric) e ferramentas de visualização. Rajashi Guha e Andrew Lang desenvolveram interfaces que comparam visualmente duplicate runs ${ }^{20}$ enquanto Bradley experimentava o jogo on-line Second Life como ferramenta para visualização de moléculas em $3 \mathrm{D}$ por seus alunos.

Motivado pela ideia de uma "ciência rápida, ciência melhor", Bradley vislumbrava na automação a possibilidade de diminuir a incidência de erros humanos e fomentar um perfil mais analítico dos pesquisadores. Por isso, cria um fluxo de trabalho (workflow) automatizado para integrar repositórios, blogs, wikis, bancos de dados, ferramentas de busca e aplicativos, aumentar a precisão dos registros, conservar a proveniência dos dados e evitar a perda de informação. No caderno aberto, logs automatizados melhoram a qualidade dos registros porque, conforme ele mesmo questiona, se você "olhar um caderno de laboratório em um laboratório típico de química orgânica - você pode realmente reproduzir todos aqueles experimentos sem conversar com o pesquisador?"21

\section{A tecnologia social}

Na proposta de Bradley, o significado de "ser cientista" ou, em outros termos, de ser um especialista, é ressignificado em vários sentidos. Em primeiro lugar, a diminuição de barreiras de entrada pela não exigência de certificações formais confere a qualquer pessoa (ou sistema automatizado), capaz de realizar contribuições relevantes, a condição de par. Nas suas palavras, "eu não me importo se [as contribuições] provêm de um alguém laureaudo com um Nobel, um precoce de 14 anos ou um bot”. ${ }^{22}$

Os intercâmbios formais entre pesquisadores não se limitam mais à comunicação de fatos científicos via artigos, mas se diversificam quantitativamente e qualitativamente. $\mathrm{O}$ formato storyless experiments exime o pesquisador do papel de autor de narrativas coerentes sobre sua pesquisa, promovendo o trânsito de uma comunicação focada nos resultados finais de experimentos, por vezes muito complexos, para uma abordagem focada em resultados parciais, independentemente de seu status - em andamento, finalizado, abandonado, favorável ou ambíguo. Já a adoção de licenças abertas permite que os pesquisadores sejam autores de contribuições sem sua conversão em proprietários de informação. Conjugadas essas características, o caderno aberto de laboratório fomenta a transição do "tudo ou nada" da cultura do segredo para uma economia de microcréditos que reconhece vários tipos de colaboração e registra a marca temporal (timestamp) de quem fez uma contribuição, quando, onde e como de maneira independente.

Nessa nova perspectiva, qualquer experimento bem documentado em um caderno aberto de laboratório deve ser considerado uma contribuição à ciência. Por isso, Bradley advoga pelo compartilhamento precoce de informações em contraposição à tendência de procrastinação dos cientistas, fomentada justamente pela expectativa da construção do fato científico. A abertura dos cadernos de laboratório estabelece um processo permanente de revisão aberta por pares, no qual potenciais colaboradores acessam a documentação detalhada de experimentos e podem verificar sua adequação ao tentar obter o mesmo conjunto de evidências 
que o autor original afirma ter produzido. Eles atuam como testadores (testers) em um novo modo de comunicação que não se baseia mais na ideia de "fontes confiáveis", mas promove a transparência e a proveniência dos dados, pois considera-se que "a verdade por inteira é mais valiosa do que um subconjunto apresentado de maneira que possa ser convenientemente confusa." ${ }^{23}$. Vale ainda lembrar que a emergência de novos padrões de descoberta com o uso de ferramentas de inteligência orientada por dados (data driven intelligence) também estabelece novos perfis profissionais. Na Química, os quimioinformatas são os profissionais que extraem significado de bases de dados abundantes e sua relevância profissional está na capacidade de manipular informação e realizar perguntas inéditas e fundamentais.

\section{As publicações líquidas de Casati, Giunglia e Marchese}

O diagnóstico de Bradley converge fortemente com a análise de Casati, Giunchiglia e Marchese ${ }^{24}$, pesquisadores oriundos da área de tecnologia de informação, que propõem a substituição do paradigma de "publicações sólidas", características do mundo impresso e analógico, por "publicações líquidas" mais adequadas à era digital.

Segundo os autores, a despeito da popularização da internet em vários campos da atividade social, a comunicação científica ainda se baseia no modelo anacrônico de "publicações sólidas", descritas como contribuições escritas, elaboradas por um círculo fechado e restrito de autores, revisadas e publicadas em um formato de artigo científico que "gravam em pedra", "cristalizam no tempo e no espaço" um conhecimento científico, tornando-o permanente e não atualizável. Eles identificam que o paradigma sólido da comunicação científica "funcionou" até então porque era a única alternativa viável no contexto préinternet, quando as plataformas tecnológicas então disponíveis não permitiam a atualização contínua dos resultados publicados em revistas científicas, além de restringir a avaliação por pares a um pequeno grupo de especialistas. Porém, este modelo é hoje considerado contraproducente, pois o produtivismo acadêmico fomenta a proliferação de "artigos incrementais" - que pouco adicionam a resultados já publicados e desperdiçam recursos na sua escritura e avaliação.

Com a noção de "publicações líquidas”"24, Casati, Giunchiglia e Marchese assinalam a necessidade de substituir o modo dominante da comunicação científica, centrada em artigos não atualizáveis, por um conjunto de contribuições que podem evoluir de maneira contínua. Assim como Bradley, eles também se inspiram nas "lições aprendidas" no desenvolvimento ágil de software Open Source, bem como nas dinâmicas de avaliação de contribuições em comunidades on-line, para propor mecanismos de revisão por pares e de atribuição de autoria, que consideram mais precisos e justos para recompensar a disseminação precoce de conhecimento científico. Isto porque reconhecem que tanto a produção científica quanto o desenvolvimento de software são “criações artísticas” complexas, cuja principal característica é a maleabilidade. Ou seja, são criações colaborativas, evolutivas, multifacetadas, multiuso, agregáveis e adaptáveis em várias direções a partir das ideias, preferências ou necessidades de um indivíduo ou grupo. O paradigma das publicações líquidas advoga por:

- Uma abordagem mais colaborativa e evolucionária da escrita de artigos com o objetivo de modificar a maneira de compartilhar o conhecimento científico e atribuir crédito aos colaboradores. No formato líquido, os trabalhos passam a ser "versionados", compilam mudanças incrementais e facilitam a colaboração entre grupos.

- Desenvolvimento de formas alternativas às publicações sólidas que ainda dominam processos de comunicação, disseminação, avaliação, reconhecimento e recuperação do conhecimento científico.

- Complementação dos métodos de revisão por pares pela incorporação de práticas de avaliação 
sociais típicas da internet, nas quais as contribuições são classificadas ou ranqueadas por membros de uma comunidade, não sendo mais restritas aqueles que exibem melhor reputação.

- Modelo de negócios mais sustentáveis que resolvam os atuais entraves econômicos do acesso ao conhecimento, mas também ofereçam novos serviços para fomentar a disseminação precoce do conhecimento, tais como as redes sociais científicas, blogs, social bookmarking, notificações de novas contribuições em uma determinada área, etc.

O paradigma líquido seria concretizado por uma nova tecnologia literária, de "objetos de conhecimento científico" (scientific knowledge objects - SKO), articulada a novos processos e papéis na produção e comunicação de conhecimento científico.

O SKO é a contrapartida digital da noção tradicional de artigo científico. Ele é um objeto de conhecimento multi-facetado e multi-propósito, composto por diferentes tipos de contúdo (texto, imagens. videos, slides, datasets, pareceres, comentários da comunidades etc) que cumpre vários objetivos de comunicação. São publicações evolucionárias e colaborativas que apresentam as múltiplas versões de uma mesma pesquisa, registrando a sua evolução. Equipada com ferramentas de controle de versão, assigna tanto o crédito quanto a responsabilidade dos colaboradores por suas contribuições. Além disso, permite que outros caminhos sejam explorados por indivíduos ou grupos "dissidentes" em um mecanismo análogo ao fork no desenvolvimento de software.

O ciclo de vida de um SKO depende da manutenção de processos que garantam sua constante evolução e fomentem a disseminação precoce do conhecimento científico em seu formato colaborativo e atualizável. Nesse sentido, incrementam-se as demandas e as oportunidades de participação da comunidade cientifica em diversas etapas da produção e comunicação do conhecimento porque as publicações líquidas expandem os papéis tradicionais de autor, leitor e revisor, antes restrito ao artigo científico, para inúmeros documentos e pessoas. Agora, os pares atuam como coautores, leitores e revisores de um artigo, de um rascunho de um artigo, de uma revisão de um artigo, de um resultado incremental de um artigo anterior, da revisão de um comentário de um artigo, do comentário sobre um comentário de um artigo e assim por diante.

Para os autores, os revisores são um tipo específico de leitor que, ao prover pareceres sobre o trabalho de outros, se tornam um tipo específico de autor. Nesse modelo, toda a comunidade científica é lançada a participar dos processos de produção, comunicação, avaliação, manutenção e consumo do conhecimento, pois os pesquisadores podem desempenhar papéis inéditos, que igualmente contribuem na criação e evolução dos Skos, tais como classificadores (taggers), bloggers, bookmarkers, agregadores de conteúdos, etc. A intenção é fomentar a produção de objetos de conhecimento de alta qualidade, porém mais leves do que as práticas dominantes de revisão por pares.

\section{A construção de uma nova cultura epistêmica}

Pelo exposto até aqui, é possível reconhecer que diversas inovações estão sendo criadas e testadas com o objetivo de promover novos modos de produzir e comunicar ciência, agora orientada pelo paradigma aberto e para ampla colaboração. Enquanto a proposta das publicações líquidas de Casati, Giunchiglia e Marchese parece não ter avançado, o caderno aberto de laboratório tem, em alguma medida, prosperado ao ponto de sinalizar a constituição de uma nova cultura epistêmica entre uma comunidade de praticantes ainda embrionária.

Através da proposta do caderno aberto de laboratório, Jean-Claude Bradley e seus colaboradores questionam os valores que o modo dominante de comunicação científica sustenta e promove, tais como segredo, individualismo e não cooperação. De modo análogo ao movimento Open Source que o inspira, o autor pretendia criar um método de desenvolvimento para a ciência - aberto, robusto e veloz - que 
aumentasse sua capacidade de inovar e melhorasse as contribuições científicas para os problemas da humanidade. Por isso, apostou em abrir o conhecimento científico em um grau mais elevado, vislumbrando a possibilidade de uma ciência menos egocêntrica (egoless science) ${ }^{25}$ em nível experimental, na qual os pesquisadores não adiam mais o compartilhamento de suas pesquisas por conta da fantasia da execução perfeita de um experimento e do medo de obter resultados questionáveis.

Nessa nova perspectiva, Bradley busca enfrentar, de maneira pragmática, as questões editoriais (mediação da comunicação do conhecimento científico por gatekeepers), jurídicas (direitos autorais e licenças livres) e técnicas (formatos abertos e tecnologias livres) necessárias para a abertura do conhecimento. Por outro lado, investe pesadamente no aspecto social, que anteriormente fazia parte da definição de conhecimento aberto $^{\text {xvi }} 26$ e que pontuava a necessidade de abrir mão não apenas das versões finais de obras, mas o seu "código-fonte" subjacente - considerado a "matéria-prima do conhecimento" e que permite analisar, modificar e elaborar obras derivadas e, acima de tudo, "evitar que informações sejam mantidas em segredo ou abandonadas em uma gaveta". ${ }^{26}$

Reconhecemos, por sua vez, múltiplas convergências entre as propostas dos cadernos abertos de laboratório e o paradigma das publicações líquidas. Ao adotar o modelo de comunicação de translação, o caderno aberto de laboratório também disponibiliza o "código-fonte" da produção científica. Esta inovação estimula uma "economia da dádiva" na qual a reputação de pesquisadores não é construída pela avaliação positiva de uma "versão compilada", o artigo, mas pelo valor do "código doado" à comunidade ampliada de pares. Além disso, fomenta processos de revisão aberta por pares que, independentemente de credenciais formais que ostentam, estão permanentemente convidados a colaborar. No entanto, conforme assinalam Casati, Giunchiglia e Marchese, o paralelismo entre desenvolvedores de software e pesquisadores tem limitações, pois enquanto o primeiro grupo compreende o reuso do código como a maneira mais eficiente de provar a importância de sua contribuição, o segundo grupo evita compartilhá-lo por receio de que suas contribuições sejam roubadas e creditadas a outros.

O caderno aberto de laboratório é uma inovação que objetiva ultrapassar o que são consideradas falhas estruturais da ciência como, por exemplo, o excesso de confiança incorporada no processo anônimo de revisão por pares na medida em que ela é projetada como um valor da própria ciência, idealizando-a como lugar dos fatos (e não de opiniões), de controle pleno das variáveis e protocolos (sem happy accidents), da escrita (contra a oralidade) e da verdade (e não de falseamentos). Visa promover excelência pela via da desconfiança - o que não significa a instituição de um estado policialesco, mas a tentativa de esclarecer que a prática científica é muito distinta das imagens de estabilidade, domínio e certeza que povoam o imaginário coletivo. Por isso, sua tecnologia literária não investe em persuadir leitores, tampouco encerrar precocemente controvérsias. O caderno aberto é o elemento central de um "ecossistema de colaboração aberta, constituído por um conjunto de ferramentas que criam uma "arquitetura da atenção" ${ }^{27}$ a serviço de uma "serendipidade projetada", cujo objetivo é "conduz[ir] a atenção do especialista certo para o problema certo no momento certo" ${ }^{27}$ de modo que seus "micro expertises latentes" respondessem just in time a uma questão pontual que bloqueia o desenvolvimento de pesquisas. A produção e a comunicação do conhecimento científico passam a ser atividades síncronas, integradas e automatizadas.

Vale ressaltar que Bradley adotava o paradigma aberto com certo pragmatismo, aproveitando as oportunidades à mão. Ele adotou, por exemplo, serviços on-line que não são ferramentas livres, ponderando a relação custo-benefício do uso de plataformas ubíquas e fáceis de usar. Esse senso de oportunidade também caracteriza sua relação com indústrias farmacêuticas no desenvolvimento aberto de drogas antimaláricas. A colaboração com agentes comerciais é possível desde que as práticas de caderno aberto e licenças livres 
sejam adotadas, pois funcionam como um mecanismo anti-propriedade que pretende impedir que novos monopólios sobre o conhecimento impeçam que o desenvolvimento científico alcance as pessoas.

Dadas as características das tecnologias material, literária e social criadas ao redor da proposta de caderno aberto de laboratório descritas, concluímos que o caderno aberto de laboratório é um dos elementos de uma nova cultura epistêmica que, ao contrário da Ciência Moderna, não tem como seu principal alicerce a construção de fatos científicos. Sua cultura epistêmica, que nomeamos "questões de prova", institui novas convenções e dispositivos que valorizam, sobretudo, a documentação minuciosa da prática laboratorial e cujos resultados devem ser considerados, no melhor dos casos, evidências sobre as quais os pesquisadores realizam uma série de suposições no processo de análise, mas nunca interpretados como incontestáveis. Sua tecnologia literária estabelece um ecossistema de colaboração aberta e fomenta a revisão aberta entre pares. A validação do conhecimento é realizada por colaboradores que atuam como testadores (testers) na medida em que acessam o seu conteúdo para questionar descrições e resultados, apontar omissões, repetir o experimento, sugerir alterações, retificações e desenvolvimentos futuros. Enfim, retroalimentar a pesquisa científica de forma aberta, contínua, sem intermediários.

\section{Referências}

1. Knorr-Cetina K. Epistemic cultures: how the sciences make knowledge. Harvard University Press, 1999.

2. Bradley JC, Lang A, Koch S, Steve; NEYLON, Cameron. Collaboration Using Open Notebook Science in Academia. In: Collaborative Computational Technologies for Biomedical Research, First Edition. John Wiley \& Sons, 2011.

3. Shapin S, Shaffer S. Leviathan and the air-pump: Hobbes, Boyle and the experimental life. Princeton University Press, 1985

4. Bradley JC. Open Notebook Science. [Internet]. Drexel COAS E-learning; 2006 set 26 [citado 2015 out 12]. Disponível em: http://drexel-coas-elearning.blogspot.com.br/2006/09/open-notebook-science.html

5. Bradley JC. Peer review in Google Age [Internet] Drexel CoAS E-Learning; 2006 fev 13 [citado em 2015 mar 18]. Disponível em http://drexel-coas-elearning.blogspot.com.es/2006/02/peer-review-ingoogleage.html

6. Bradley JC. Shining a light on chemical properties with Open Notebook Science and open strategies. Lecture at ACS Symposium on August 20, 2012 [Internet] [citado em 2015 fev 5]. Disponível em https://www.youtube.com/watch?v=7mK6SY-jXqk

7. (Bradley, Aug, 20, 2012)

8. Bradley JC. Science is about mistrust [Internet] UsefulChem; 2007 out 04 [citado 2015 mar 18]. Disponível em http://usefulchem.blogspot.com.es/2007/10/science-is-about-mistrust.html

9. Bradley JC. Opening up and sharing [Internet] Chemistry World; 2013 abril 18 [citado em 2014 fev 20]. Disponível em http://www.rsc.org/chemistryworld/2013/04/open-science-chemistry-sharinginformation

10. Bradley JC. Blogger as lab notebook [Internet] Drexel CoAS E-Learning Blog; 2006 fev 9 [citado 2015 mar 18]. Disponível em http://drexel-coas-elearning.blogspot.com.es/2006/02/blogger-as-labnotebook. $\underline{\mathrm{html}}$

11. Bradley JC. Interview With Jean-Claude Bradley. The Impact of Open Notebook Science by Richard Poynder [Internet]; Information Today; 2010 set [citado em 2014 jan 24]. Disponível em http://www. infotoday.com/it/sep10/Poynder.shtml\#top

12. Bradley JC. Science is about mistrust [Internet] UsefulChem; 2007 out 04 [citado 2015 mar 18]. Disponível em http://usefulchem.blogspot.com.es/2007/10/science-is-about-mistrust.html

13. Bradley JC. There are no facts: my position at NSF eChem workshop [Internet] UsefulChem; 2008 out 26 [citado em 2015 dez 15]. Disponível em http://usefulchem.blogspot.com.br/2008/10/there-are-nofacts-myposition-at-nsf.html

14. Latour B. A ciência em ação: como seguir cientistas e engenheiros sociedade a fora. São Paulo: UNESP, 2000. 
15. Latour B. Science in Action: How to Follow Scientists and Engineers Through Society, Harvard University Press, 1987.

16. Bradley JC. Scientific American Science 2.0 Article. [Internet] UsefulChem; 2008 jan 9 [citado em 2014 ago 12]. Disponível em http://usefulchem.blogspot.com.es/2010/02/funding-agencies-andopen-science. $\underline{\mathrm{html}}$

17. Bradley JC. C\&E News Article on Writing [Internet]. UsefulChem; 2007 ago 13 [citado em 2015 ago 18]. Disponível em http://usefulchem.blogspot.com.br/2007/08/c-news-article-on-writing.html

18. Bradley JC. Open Notebook Science Claims and Logos [Internet] UsefulChem; 2009 fev 24 [citado em 2013 nov 17]. Disponível em http://usefulchem.blogspot.com.br/2009/02/open-notebook-scienceclaimsand-logos.html

19. Bradley JC. UsefulChem on ChemSpider [Internet] UsefulChem; 2008 abril 27 [citado em 2016 jan 21]. Disponível em http://usefulchem.blogspot.com.es/2008/04/usefulchem-onchemspider.html

20. Bradley JC. Google Visualization API on ONS solubility data [Internet]; UsefulChem; 2008 nov 06 [citado 2015 out 22]. Disponível em http://usefulchem.blogspot.com.es/2008/11/googlevisualization-api-on-ons. $\underline{\mathrm{html}}$

21. Bradley JC. Modularizing Results and Analysis in Chemistry [Internet]; UsefulChem; 2008 jan 3 [citado em 2015 out 20]. Disponível em http://usefulchem.blogspot.com.br/2008/01/modularizing-results-andanalysis-in.html

22. Bradley JC. Remote Controlled Labs [Internet]; UsefulChem; 2006 dec 12 [citado em 2015 out 20] Disponível em http://usefulchem.blogspot.com.br/2006/12/remote-controlled-labs.html

23. Bradley JC. Funding Agencies and Open Science [Internet]; UsefulChem; 2010 fev 28 [citado em 2009 fev 12]. Disponível em http://usefulchem.blogspot.com.es/2010/02/funding-agencies- andopen-science. $\underline{\mathrm{html}}$

24. Casati F, Giunchiglia F, Marchese M. Liquid Publications: Scientific Publications meet the Web. Technical Report DIT-07-073. December 2007

25. Bradley JC. Is the Human Ego good for Science? [Internet]; UsefulChem; 2009 abr 07 [citado 2015 dez 15]. Disponível em http://usefulchem.blogspot.com.br/2009/04/is-human-ego-good-for-science.html

26. Pollock R. The Three Meanings of Open [Internet] Open Knowledge [citado em 2014 jan 13]. Disponível em http://blog.okfn.org/2004/06/21/the-three-meanings-of-open

27. Nielsen M. Reinventing discovery: the new era of networked science. Princeton: Princeton University Press, 2011. 